Aus der chirurgischen Universitätsklinik in Bonn

(Direktor: Geh. Rat Prof. Garrè).

\title{
Hernia uteri inguinalis bilateralis.
}

\author{
Von Dr. M. Makkas, Assistenzarzt der Klinik.
}

Wenn schon Brüche mit der Gebärmutter als Inhalt zu den großen Seltenheiten gehören, so scheint der Fall von doppelseitiger Uterushernie, den ich Gelegenheit hatte zu beobachten und zu operieren, ein Unikum zu sein, so daß seine Mitteilung gerechtfertigt erscheint. Die Krankengeschichte meiner Patientin ist folgende:

Frau Emma Sch., 47 Jahre alt. Patient will früher immer gesund gewesen sein. Menstruation regelmäßig! Keine Kinder. Seit 2-3 Jahren bemerkt die Frau eine kleine Anschwellung in der linken Leistengegend, die beim Arbeiten heraustritt und bei Ruhelage verschwindet. Seit etwa I4 Tagen geht die Geschwulst nicht mehr zurück. Schmerzen haben nie bestanden. Kein Erbrechen, Stuhl regelmäßig. Keine Beschwerden beim Urinieren.

Die Untersuchung ergab eine mittelgroße, kräftige Frau in gutem Ernährungszustand. Innere Organe gesund, Urin frei von Eiweiß und Zucker.

In der linken Leistengegend findet sich ein ovaler, über gänseeigroßer Tumor, der schräg von oben außen, nach unten innen verläuft und das große Labium ausfüllt. Die Oberfläche des Tumors ist glatt, die Konsistenz prall elastisch, der Perkussionsschall über der Geschwulst gedämpft. Der Tumor läßt sich nicht in die Bauchhöhle reponieren, auch durch Druck nicht verkleinern.

Rechts findet sich ein über wallnußgroßer Tumor, der ebenfalls bis in die große Schamlippe herabsteigt. Ein kleinfingerdicker Strang geht vom Tumor in den äußeren Leistenring. Der Tumor läßt sich leicht in den Leistenkanal zurückschieben, um beim Nachlassen des Druckes sofort wieder herauszutreten.

Auf Grund dieses Befundes wurde links eine irreponible Leistenhernie mit Netzinhalt diagnostiziert, rechts nahmen wir an, daß es sich um einen gestielten, freibeweglichen Netzklumpen handelte. 
I5. II. r910. Operation in Lokalanästhesie mit $1 / 2$ Proz. Novocain-Suprareninlösung.

L inke S e it e. $8 \mathrm{~cm}$ langer Längsschnitt über der Geschwulst. Dieselbe wird aus dem großen Labium ausgeschält, so daß sie gestielt aus dem äußeren Leistenring heraushängt. Auch nach Spaltung der Aponeurose des Obliquus externus läßt sich der Bruchinhalt nicht reponieren. Jetzt folgt Eröffnung des Bruchsackes an seiner vorderen Wand. Als Bruchinhalt findet sich das Ovarium, die Tube und der Uterus. Das Ovarium ist oval, flach, kuchenförmig, seine Oberfläche narbig glänzend. Es ist etwa $6 \mathrm{~cm}$ lang, $4 \mathrm{~cm}$ breit und $2 \mathrm{~cm}$ dick. Die Konsistenz ist schlaff. Neben dem Ovarium liegt geschlängelt die schlanke, normal aussehende, $7-8 \mathrm{~cm}$ lange Tube, deren freies Ende gut entwickelte Fimbrien zeigt. Nach Anhebung des Eierstockes sieht man an der Innenseite des Bruchsackes einen kleinen hornförmig gebogenen Uterus von etwa $6 \mathrm{~cm}$ Länge. Das Horn des ganz im Bruche liegenden Uterus sieht nach vorn außen, der cervikale Teil nach hinten innen. Der Peritonealüberzug des Uterus geht direkt in den Bruchsack über, ebenso ist das Ligamentum latum unterhalb des Ovarium nicht mehr vorhanden, sondern seine zwei Blätter sind auseinandergeschoben und bilden einen Teil des Bruchsackes. Das Ligamentum ovarii ist ziemlich lang. Das Ligamentum rotundum geht von der Außenseite des Bruchsackes, wo der Uterus extraperitoneal liegt, aus. Von der konvex abgerundeten rechten Kante des Uteruskörpers geht ein bleistiftdicker Strang nach rechts ins Abdomen. Er bildet den oberen Rand einer, von der rechten Uteruskante abgehenden, quer durch das Becken ziehenden Peritonealduplikatur und ist im Inneren des Abdomens fixiert, so da $B$ er sich nicht herausziehen läßt. Ein rechtsseitiges Ovarium läßt sich nicht abtasten. Vor der rudimentären Portio des Uterus fühlt man ein weiches, verschiebliches Gewebe, was den Verdacht erweckt, daß es sich um die Blase handeln könnte, doch überzeugt man sich durch Einführen eines Metallkatheters in die Blase, daß dieselbe im kleinen Becken liegt. Nach Reposition von Uterus, Ovarium und Tube, werden die Ränder der Peritonealöffnung genäht und dann die Bruchpforte, durch Naht des Obliquus internus an das Poupartsche Band, geschlossen. Aponeurosenaht, Hautnaht.

Rechte Seite. $7 \mathrm{~cm}$ langer Schrägschnitt. Freilegung des Bruchsackes und Eröffnung desselben. Es zeigt sich nun, daß der im Bruchsack gefühlte walnußgroße Körper, der kleine hornförmige rechte Uterus ist. Er ist kleiner als der linke, etwa $4^{1 / 2} \mathrm{~cm}$ lang, atrophisch, mit kaum angedeuteter Cervix. Er liegt auch in der Bruchsackwand, die durch Entfaltung der Blätter des Ligamentum latum entstanden ist. Neben dem Uterus liegt im Bruchsack die normal aussehende, geschlängelte Tube. Nach Erweite- 
rung des Bruchsackhalses wird aus der Bauchhöhle das rechte Ovarium herausluxiert. Es ist walnußgroß, kugelig hart, von kleinen Cysten durchsetzt. Von der linken medialen Uteruskante zieht eine Peritonealduplikatur in die Tiefe des Abdomens, an deren oberen Rand ein bleistiftdicker Strang verläuft. Der Strang ist $7-8 \mathrm{~cm}$ lang und endigt an einem harten, beweglichen, links von der Mittellinie liegenden Körper, der nach Lage und Form dem reponierten linken Uterus entspricht. Nach Reposition des Bruchinhaltes VerschluB des Peritonealloches durch Naht. SchluB der Bruchpforte.

Der postoperative Verlauf war glatt, so daß Patient am 8. Tage das Bett verlassen konnte. Nach der Operation gibt die Kranke auf Befragen zunächst bestimmt an, daß die Periode immer regelmäßig gewesen sei; Schmerzen im Bauch während der Periode sollen nie bestanden haben. Erst als wir durch eine gynäkologische Untersuchung eine Atresie der Vagina feststellten, bequemte sie sich zu dem Geständnis, daß sie nie menstruiert gewesen sei. Allerdings sollen auffallenderweise auch niemals Molimina menstrualia bestanden haben.

Gynäkologischer Befund (Prof. Reifferscheid): Weiblicher Habitus. Mammae schlecht entwickelt, Beckenmaße normal, äußere Genitalien normal. Kleine Labien auffallend klein. Scheideneingang breit, von einzelnen Schleimhauterhebungen umgeben, die als Reste des Hymen angesprochen werden können. Mit dem Finger kommt man in einen Blindsack, der sich etwa $6-7 \mathrm{~cm}$ weit einstülpen läßt. Cervix oder Portio nicht zu fühlen. Auch per rectum gelingt es nicht, die Uterushörner und die Ovarien abzutasten, wobei die Empfindlichkeit der noch frischen Operationsnarben hinderlich ist.

Es handelt sich also in unserem Falle um eine Mißbildung in Form des Uterus bipartitus, dessen beide Hälften jederseits in eine Inguinalhernie eingetreten waren. Die Doppelseitigkeit der Uterushernie bedingt die große Seltenheit des Falles; in der mir zugänglichen Literatur konnte ich keinen ähnlichen Fall finden. Einfache inguinale Uterushernien sind nicht so selten, in der Literatur sind 27 Fälle bekannt ${ }^{1}$ ).

I) Fälle von Maret, Lallemant, Chopart, Olshausen, Leopold, Schwartz, Brohl, Legneu, Diederich, Perondi, Krug, Laurent, Rouffart, Brunner, Maschka, Bylicki, Pol, Sennert, Saxtorph, Lédesma, Rektorzik, Scanzoni, v. Winckel-Eisenhart, Rosanoff, Defontaine, Simon and $\mathrm{Sabitier}$, Gowey. Vgl. die Zusammenstellungen von $\mathrm{Con}$ a d Brunncr, Hernionogische Beobachtungen. Beiträge zur klin. Chirurgie I889, Bd. 4. Richard Bir n b a $\mathrm{m}$, Beitrag zur Kenntnis der Hernia uteri ingui- 
Es handelte sich 16 mal um Hernien des nicht graviden und I I mal des graviden Uterus.

Auffallend häufig findet man unter den Fällen von Uterushernien Mißbildungen der Genitalien, meistens Uterus bicornis oder bipartitus, sehr oft auch Atresie der Vagina. Küstner fand unter I I Fällen von Hysterocele des nicht graviden Uterus 8 mal Bildungsanomalien desselben, unter I I graviden Fällen 2 mal. Die Häufigkeit der Mißbildungen des Uterus in Fällen von Hysterocele spricht mit großer Wahrscheinlichkeit für einen ätiologischen Zusammenhang beider. Besonders im doppelten Uterus muß durch die Annäherung der Uterushälften an den inneren Leistenring das Hinaustreten einer oder auch beider Hälften, wie in unserem Falle, in eine Hernie begünstigt werden. Klob hebt mit Recht hervor, daß die allmähliche Vergrößerung eines bestehenden Bruchsackes auf Kosten der das Ligamentum latum konstituierenden Peritonealduplikatur geschieht, und daß dadurch der Uterus zunächst an die Bruchpforte gezogen und schließlich in dieselbe, ja durch dieselbe zu treten genötigt ist. Dieser Vorgang wird auch durch unseren Fall bestätigt, wo die Blätter des Ligamentum latum unterhalb des Ovarium auseinander geschoben waren und einen großen Teil der Bruchsackwand bildeten. Der Bruchsack kann anscheinend ein angeborener, durch Offenbleiben des Diverticulum $\mathrm{Nuckii}$ entstandener sein, wie die Fälle von Uterushernien bei Säuglingen beweisen (Fall Laurent, Defontaine), oder ein in späterem Alter erworbener. $Z \mathbf{u}$ letzteren muB auch unser Fall gerechnet werden. In den meisten Fällen scheint es, daß erst das Ovarium in den Bruchsack ausgetreten ist, und durch Zug desselben der Uterus nachfolgte. Auch in unserem Falle scheint die linke Uterushälfte durch $\mathrm{Zug}$ des Ovarium in den Bruch herausgetreten $z \mathbf{u}$ sein, denn bei ausgetretenem Uterus war die Bruchpforte so eng, daß das ziemlich große Ovarium nicht hätte heraustreten können. Rechts fällt diese Möglichkeit weg, da das Ovarium in der Bauchhöhle sich fand, und so groß und hart war, daß ein Heraustreten desselben in den

nalis und der histologischen Veränderungen verlagerter Ovarien. Berliner klin. Wochenschr. I905, S. 632, K üs t in e r, Hernie uteri im Handbuch der Gynäkologie von Veit I907, 2. Aufl., I. Bd., S. 407. 
Bruch nicht möglich gewesen wäre. Durch Zug und Zerrung der zwei Uterushälften in entgegengesetzter Richtung entfernten sich diese nun immer weiter voneinander; so erklärt sich die relativ große Breite der die zwei Uterushörner verbindenden Peritonealduplikatur.

In anderen Fällen wurde als ätiologisches Moment eine adhäsive Peritonitis angeführt, die zu Verlötungen des Uterus mit anderen Eingeweiden, Netz oder Darmschlingen geführt hatte. In diesem Falle soll der Uterus sekundär durch Zug der in den Bruchsack geratenen Eingeweide verlagert werden. Bei unserer Patientin kann diese Art der Entstehung nicht in Betracht gezogen werden.

Was die Form der Uterusmißbildung in unserem Falle anlangt, so glauben wir, daß es sich um eine aus dem zweiten Monat des Fötallebens stammende handelt, und zwar, wenn wir uns an die Einteilung von Winckel1) halten, entweder um einen Uterus solidus rudimentarius duplex cum vagina solida oder um einen Uterus rudimentarius, partim excavatus duplex, cumvagina solida. Jedenfalls scheint das Fehlen von Molimina menstrualia gegen das Vorhandensein einer funktionsfähigen Uterusschleimhaut zu sprechen. Leider haben wir eine Probeincision des Uterus unterlassen, die uns über das Bestehen eines Lumens aufgeklärt hätte.

Die Diagnose in unserem Falle wurde erst bei der Operation gestellt, da die gynäkologische Untersuchung vor der Operation unterlassen wurde. Im allgemeinen ist die Diagnose nicht schwierig. Als subjektives Symptom von Ovarial- und Uterushernien werden in erster Linie die periodisch auftretenden Schmerzen im Bauch angeführt. Das gilt allerdings nur für menstruierende Frauen. In Fällen von rudimentärem Uterus und fehlender Menstruation wird dieses Symptom vermißt. Dagegen scheinen die objektiven Symptome in sehr vielen Fällen so eindeutig gewesen zu sein, daß die Diagnose ohne Schwierigkeit gestellt werden konnte. Am leichtesten war sie offenbar bei

I) M e $\mathrm{nge}$, Bildungsfehler der weiblichen Genitalien in Veits Handbuch der Gynäkologie I9IO, Bd. 4 . 
den Fällen, in denen ein gravider Uterus als Bruchinhalt vorlag. Das Ausbleiben der Menses, das schnelle Anwachsen des Tumors, die Kindesbewegungen, die Palpation des Fötus und die Auskultation der Herztöne, hat in den meisten Fällen die richtige Diagnose ermöglicht. Bei nicht graviden Fällen, wenn die periodisch auftretenden Schmerzen in der Geschwulst fehlten, wurde die Diagnose nur in wenigen Fällen richtiggestellt. Jedenfalls muß eine Bildungsanomalie der Vagina, Verschluß oder Fehlen derselben bei einer Frau mit einem härteren Körper in einer Leistenhernie immer den Verdacht einer Ovarial- oder Uterushernie erwecken. Wir haben ja gesehen, daß dieser Bildungsfehler sehr oft bei Uterushernien gefunden wurde. Die Rektaluntersuchung würde in einem solchen Falle die Bestätigung der Diagnose bringen können.

In den nicht graviden Fällen wird die operative Therapie in der Regel konservativ bleiben, insofern als Uterus und Adnexe einfach reponiert werden und die Bruchpforte geschlossen wird. Die Exstirpation des Uterus wird nun in bestimmten Fällen indiciert sein. Bei gravidem Uterus, wenn die Diagnose rechtzeitig gestellt werden kann und die Reposition des Bruches nicht gelingt, wird der künstliche Abort ( $\mathrm{Schultze}$ ) einzuleiten sein. Bei ausgetragener Schwangerschaft dagegen ist die Entbindung durch den Kaiserschnitt das richtige Verfahren. 